\title{
Risk-Minimizing Hedging for Indexed Stock Options under Jump-Diffusion Processes
}

\author{
Jianhua Guo \\ Business School, University of Shanghai for Science and Technology \\ 516 Jungong Road, Shanghai 200093, China \\ E-mail: jhguo888@163.com \\ Qingxian Xiao \\ Business School, University of Shanghai for Science and Technology \\ 516 Jungong Road, Shanghai 200093, China
}

Received: June 7, 2011

Accepted: August 1, 2011 Published: December 1, 2011

doi:10.5539/ijbm.v6n12p232

URL: http://dx.doi.org/10.5539/ijbm.v6n12p232

\begin{abstract}
With conditional mean square error of hedging cost process as risk measure, this paper presents risk-minimizing hedging for indexed stock options under jump diffusion processes. Firstly, the cost process of hedging with risk-minimizing criterion is testified to be a martingale. Then, the explicit optimal strategy is given using backward recursive method. Lastly, an exemplification based on China Stock Markets is given as an example to illuminate the relationship between underlying asset positions and option's maturity horizon and position adjusting frequency.
\end{abstract}

Keywords: Indexed stock options, Hedging, Risk-minimizing Criterion, Jump-diffusion process

\section{Introduction}

Hedging is one of major issues in both theoretical and applied finance and investors try to reduce the sensitivity of their portfolios to the fluctuations of the market by hedging. In their seminal paper, Black and Scholes(1973) showed that a European call option can be replicated by indirectly constructing a self-financing portfolio only consisting of the underlying asset and the other riskless asset. In practice, their model of asset price generates a complete market in which all contingent claims are attainable and investor's position must be continuously adjusted. However, many empirical approach results demonstrate that many practical financial markets are incomplete, and in such markets, any claims can not be replicated by self-financing strategy, furthermore, if only discrete hedging times are allowed, acquiring a risk-free position at each moment is impossible. Thus, there will be difference between the terminal trading portfolio and the contingent claims by self-financing strategy, or else, some cash flows have to be induced or withdrawn to continue such a perfect replication strategy, which means that additional costs occur over the lifetime of the financial derivatives that has to be hedged. So, how to measure financial risk is the first question for hedging and the 'optimal' hedging strategy must be chosen to minimize such a particular measure of risk.

Different criteria for hedging can be found in literature. Follmer and Sondermann (1986) firstly put forward the risk-minimizing hedging criterion. By relaxing self-financing constraint, they introduced the conditional mean square error of hedging cost process as risk measuring. In the case where the price process is a martingale under probability $P$, it was shown that there exists a unique risk-minimizing strategy which can be computed by using the Kunita-Watanabe projection theorem. Compare to Follmer and Sondermann's working with continuous time model and original martingale case, the risk-minimizing hedging approach was subsequently transformed to discrete time framework by Follmer and Schweizer (1989) without the constraint of martingale price processes and they obtained recursion formulas describing the optimal strategies.

Under the risk-minimizing hedging criterion, the price processes of underlying assets need to be martingales, but with respect to the original probability measure, the underlying assets price processes are practically semi-martingales, which determine that the risk-minimizing strategy might not exist. Thus, based on risk-minimizing method, Schweizer (1991) proposed the concept of locally risk-minimizing hedging criterion, 
which aimed at minimizing the variance of the cost process of non-self-financing hedges for price processes which are only semi-martingales, and showed that this criterion was similar to performing risk-minimization using the so-called minimal martingale measure, see also Schweizer (1995) and Schweizer (1999).

Different from the above two hedging approaches, Coleman et al (2003) put forward the discrete hedging method under piecewise linear risk minimization, which aimed at minimizing the absolute incremental cost between adjacent rebalancing moments. Coleman et al illustrate that, by generating synthetic paths for the asset price, the piecewise linear risk minimization may lead to smaller average total hedging cost and risk, as has also been shown that the choice of measurement for incremental cost is important in incomplete markets, for example, $\mathrm{L}_{1}$ and $\mathrm{L}_{2}$ norms can lead to significantly different trading strategies. However, there is no analytic solution to the piecewise linear local risk-minimization problem.

Literature in risk-minimizing and local risk-minimizing hedging mainly focused on traditional European options. In practice, these methods may also be used for other kinds of derivatives. For example, Schiefner (2002) used the method of risk minimization in discrete time to find the optimal hedging strategy for a financial product with a general cash flows depending on the existing assets in the economy which are characterized by price and cash flow processes. Setting out by explaining the main ideas in a simple discrete time framework and then moving on to a model with continuous time trading, Møller (2004) described the theory of risk-minimization in more detail and reviewed some applications from the insurance literature. Poulsen et al (2009) researched the risk-minimizing hedging problem when risky asset price evolved as stochastic volatility process.

Relative to traditional Option, Scholars put forward a kind of Indexed-Stock Option. The so-called Indexing Idea was firstly put forward by Ubelhart (1981) as an incentive mechanism to managers. Afterward, based on the Indexing Idea, Akhigbe et al(1996) propose an executive option, they defined the market performance as a benchmark and constructed the exercise price of option as a variable $K_{T}=S_{0} I_{T} / I_{0}$, which depends on market performance $I_{T}$. One of strong point of Indexed-Stock Option is that the indexed option pays off only when the firm's stock price exceeds a specified moving 'benchmark' stock price, when traditional stock options with fixed exercise price violate a basic proposition that contracts should insure agents against uncertainty generated by common factors beyond their control, while retaining controllable, idiosyncratic effects to provide the appropriate incentives. As an executive option, the indexed option solves this problem by filtering out the benchmark's performance, which captures the common uncertainty an executive faces. The option payoff depends on the firm's performance relative to the benchmark, and can be positive in down markets and zero in up markets.

Up to now, indexed-derivative investment is at start-up period in China, and as an investment instrument, indexed stock option may be confronted with more risk than traditional option. Still, from its emergence on, the incentive mechanism of indexed stock option has been researched by many scholars, including Calvet(2006), CHEN(2002), Jin-Chuan Duan(2005), Shane(2000) and ZHONG(2005). However, we don't find any literature about hedging for indexed stock options.

In this paper, assuming the underlying asset price evolving as a jump-diffusion process, we set the exercise price as $K_{T}=S_{0} I_{T} / I_{0}$ and attempt to study the problem of risk-minimizing hedging of indexed stock option in a discrete time framework.

The remainder of this paper is organized as follows. In the next section, we discuss the design of market model and setup of the indexed option's hedging problem. In Section 3, we present explicit risk-minimizing strategies for the hedging problem, and empirical analysis based on China Stock Markets is proposed in Section 4, while discuss and conclude in Section 5.

\section{Market Model and Hedging Problem}

Let $(\Omega, F, P)$ be a complete probability space with filtration $F=\left(F_{t}\right)_{t \in[0, T]}$, we assume that the firm's stock price $S=\left(S_{t}\right)_{t \in[0, T]}$ and the index $I=\left(I_{t}\right)_{t \in[0, T]}$ model on jump diffusion processes specified by the following equations

$$
\begin{array}{r}
d S_{t}=S_{t^{-}}\left(\mu d t+\sigma d w_{t}+q d N_{t}\right) \\
d I_{t}=I_{t^{-}}\left(\mu^{(I)} d t+\sigma^{(I)} d w_{t}^{(I)}+q^{(I)} d N_{t}^{(I)}\right)
\end{array}
$$

Where $w_{t}$ and $w_{t}^{(I)}$ are standard Brownian motion, and $t$ is time. And we can interpret $\mu$ and $\mu^{(I)}$ as the 
expected returns on the stock and the index, while $\sigma$ and $\sigma^{(I)}$ as the instantaneous volatility rates. $N_{t}$ and $N_{t}^{(I)}$, independent with $w_{t}$ and $w_{t}^{(I)}$, are Poisson processes respectively with Poisson strength $\lambda$ and $\lambda^{(I)} \cdot q\left(q^{(I)}\right)>-1$ denotes the amplitude of stock (index) price jump.

Let $B=\left(B_{t}\right)_{t \in[0, T]}$ be riskless asset price process, just as following

$$
d B_{t}=r B_{t} d t, r \text { is riskless interest rate }
$$

Definition 1 Call $\varphi=(\vartheta, \delta)$ an investment strategy, when

$$
V_{t}(\varphi):=\vartheta_{t} S_{t}+\delta_{t} B_{t} \in L^{2}(P), t=0,1, \cdots, T,
$$

Where $\vartheta_{t} S_{t} \in L^{2}(P)$ and $\delta_{t}$ is an adapted process, $V(\varphi)$ is the valuation process of strategy $\varphi=(\vartheta, \delta),\left(\vartheta_{t}, \delta_{t}\right)$ denote the stock and riskless asset positions held at time $t$.

Definition 2 The cost process corresponding with strategy $\varphi=(\vartheta, \delta)$ satisfies

$$
\begin{aligned}
& C_{0}(\varphi)=V_{0}(\varphi)=\vartheta_{0} S_{0}+\delta_{0} B_{0} \\
& C_{t}(\varphi):=V_{t}(\varphi)-\sum_{j=1}^{t} \vartheta_{j-1} \Delta S_{j}-\sum_{j=1}^{t} \delta_{j-1} \Delta B_{j}, t=1, \cdots, T
\end{aligned}
$$

Where $\Delta S_{j}=S_{j}-S_{j-1}$ (respectively, $\Delta B_{j}=B_{j}-B_{j-1}$ ) denotes the stock price (respectively, the bond price) increment.

If denoting $\Delta C_{t}(\varphi):=C_{t}(\varphi)-C_{t-1}(\varphi)$, then

$$
\Delta C_{t}(\varphi)=\Delta V_{t}(\varphi)-\vartheta_{t-1}\left(S_{t}-S_{t-1}\right)-\delta_{t-1}\left(B_{t}-B_{t-1}\right)
$$

Definition 3 The risk process corresponding with strategy $\varphi=(\vartheta, \delta)$ is defined to be

$$
R_{t}(\varphi):=E\left[\left(C_{T}(\varphi)-C_{t}(\varphi)\right)^{2} \mid F_{t}\right], t=0,1, \cdots, T
$$

Definition 4 Assuming $\varphi=\left\{\left(\vartheta_{t}, \delta_{t}\right)\right\}, t=\{0,1, \cdots, T\}$ be a trading strategy, if any given admissible strategy $\varphi^{\prime}=\left\{\left(\vartheta_{t}^{\prime}, \delta_{t}^{\prime}\right)\right\}$ satisfies, (1) $V_{T}(\varphi)=V_{T}\left(\varphi^{\prime}\right) P-a . s$, (2) Given $s \leq t, \vartheta_{s}=\vartheta_{s}^{\prime}$, (3)Given $s<t, \delta_{s}=\delta_{s}^{\prime}$, there is $R_{t}(\varphi) \leq R_{t}\left(\varphi^{\prime}\right) \quad P-a . s ., t=0,1, \cdots, T$, then we call $\varphi=(\vartheta, \delta)$ a risk-minimizing strategy.

Definition 5 Call $\varphi=\left\{\left(\vartheta_{t}, \delta_{t}\right)\right\}, t=\{0,1, \cdots, T\}$ a mean-self-financing strategy, if the cost process $\left(C_{t}, t \in[0, T]\right)$ is a martingale, i.e. $E\left[C_{T}(\varphi) \mid F_{t}\right]=C_{t}(\varphi)$

Now, suppose an investor has written a short position share of indexed stock option with exercise price $K_{T}=S_{0} I_{T} / I_{0}$ and $T$ maturity horizon at initial time 0 , and she attempts to hedge for this option with the underlying stock and the other riskless asset, e.g., the National Debt, under the constraint of $V_{T}(\varphi)=H_{T}=\left(S_{T}-K_{T}\right)^{+}$and with minimum expected cost increment $E\left[\left(C_{T}-C_{t}\right)^{2} \mid F_{t}\right]$, she can constructs the hedging model as following

$$
\left\{\begin{array}{l}
\min _{\left(\vartheta_{t}, \cdots, \vartheta_{T-1}\right)} E\left[\left(C_{T}(\varphi)-C_{t}(\varphi)\right)^{2} \mid F_{t}\right] \quad t=0,1, \cdots, T-1 \\
\text { s.t. } \quad V_{T}(\varphi)=H_{T}
\end{array}\right.
$$

\section{Solutions}

\subsection{Parameter-estimating of the Price Processes}

According to (1), there is

$$
y_{t}=\ln \left(S_{t} / S_{t-1}\right)=\mu-\frac{\sigma^{2}}{2}+\sigma \varepsilon_{t}+Z_{t} J_{t}
$$

Where $\varepsilon_{t}=w_{t}-w_{t-1} \sim N(0,1), Z_{t}=N_{t}-N_{t-1} \sim p(\lambda), J_{t}:=\ln (1+q) \sim N\left(\mu_{J}, \sigma_{J}^{2}\right)$, and $\varepsilon_{t}$ is independent of $Z_{t}$. The independent increment quality of the Brownian motion and Poisson process indicates that $\left\{y_{1}, \cdots, y_{T}\right\}$ is an independent and identical distribution sequence with conditional probability density 
function and likelihood function just as following

$$
\begin{aligned}
& p\left(y_{t} \mid Z_{t}=k\right)=\frac{1}{\sqrt{2 \pi\left(\sigma^{2}+k \sigma_{J}^{2}\right)}} \exp \left\{-\frac{\left[y_{t}-\left(\mu-\frac{\sigma^{2}}{2}+k \mu_{J}\right)\right]^{2}}{2\left(\sigma^{2}+k \sigma_{J}^{2}\right)}\right\} \\
& L\left(y_{1}, \cdots, y_{T}\right)=\prod_{t=1}^{T} p\left(y_{t}\right)=\prod_{t=1}^{T}\left\{\sum_{k=0}^{\infty}\left[\frac{\lambda^{k} e^{-\lambda}}{k !} p\left(y_{t} \mid Z_{t}=k\right)\right]\right\} \\
& =\prod_{t=1}^{T} \sum_{k=0}^{\infty}\left[\frac{\lambda^{k} e^{-\lambda}}{k !} \frac{1}{\sqrt{2 \pi\left(\sigma^{2}+k \sigma_{J}^{2}\right)}} \exp \left\{-\frac{\left(y_{t}-\left(\mu-\frac{\sigma^{2}}{2}+k \mu_{J}\right)\right)^{2}}{2\left(\sigma^{2}+k \sigma_{J}^{2}\right)}\right\}\right]
\end{aligned}
$$

As for Poisson jump, when $\Delta t \rightarrow 0$, there are $p(N(\Delta t)=1)=\lambda \Delta t+o(\Delta t)$ and $p(N(\Delta t)=0)=1-\lambda \Delta t+o(\Delta t), p(N(\Delta t) \geq 2)=o(\Delta t)$.In this paper, we estimate parameters taking advantage of high frequency historic data with time interval $\Delta t=5$ minutes, during which, it is reasonable to think there is not more than one price jump happen, i.e., $p\left(J_{t}=1\right)=\lambda, p\left(J_{t}=0\right)=1-\lambda$, thus, (11) may be substituted for

$$
\begin{aligned}
& L\left(y_{1}, \cdots, y_{T}\right) \\
& =\prod_{t=1}^{T} \sum_{k=0}^{1}\left[\frac{\lambda^{k} e^{-\lambda}}{k !} \frac{1}{\sqrt{2 \pi\left(\sigma_{S}^{2}+k \sigma_{J}^{2}\right)}} \exp \left\{-\frac{\left[y_{t}-\left(\mu_{S}-\frac{\sigma_{S}^{2}}{2}+k \mu_{J}\right)\right]^{2}}{2\left(\sigma_{S}+k \sigma_{J}^{2}\right)}\right\}\right] \\
& =\prod_{t=1}^{T} \frac{e^{-\lambda}}{\sqrt{2 \pi}}\left[\frac{1}{\sqrt{\sigma_{S}^{2}}} \exp \left\{-\frac{\left(y_{t}-\left(\mu_{S}-\frac{\sigma_{S}^{2}}{2}\right)\right)^{2}}{2 \sigma_{S}^{2}}\right\}+\frac{\lambda}{\sqrt{\left(\sigma_{S}^{2}+\sigma_{J}^{2}\right)}} \exp \left\{-\frac{\left[y_{t}-\left(\mu_{S}-\frac{\sigma_{S}^{2}}{2}+k \mu_{J}\right)\right]^{2}}{2\left(\sigma_{S}+k \sigma_{J}^{2}\right)}\right\}\right]
\end{aligned}
$$

According to (12) and refer to Cyrus A. Ramezani et al(2007), the maximum likelihood estimated values of all parameters in (1),similarly in (2), would be acquired by MLE method.

\subsection{Risk-minimizing hedging strategy}

\section{Proposition 1}

Any given risk-minimizing strategy $\varphi=(\vartheta, \delta)$ is a mean-self-financing strategy.

Proof

Refer to Schiefner L.(2002) and Follmer H, Sondermann D.(1986), For any fixed $t \in\{0,1, \cdots, T-1\}$, $\operatorname{let} \varphi^{\prime}=\left(\vartheta^{\prime}, \delta^{\prime}\right)$ satisfy

$$
\vartheta^{\prime}:=\vartheta \text { and } \delta_{s}^{\prime}:=\delta_{s} \text { when } s \neq t \text {, and } \delta_{t}^{\prime} B_{t}:=E\left[C_{T}(\varphi)-C_{t}(\varphi) \mid F_{t}\right]+\delta_{t} B_{t}
$$

Then

$$
\begin{aligned}
V_{t}\left(\varphi^{\prime}\right) & =V_{t}(\varphi)+B_{t}\left(\delta_{t}^{\prime}-\delta_{t}\right)=V_{t}(\varphi)+E\left[C_{T}(\varphi)-C_{t}(\varphi) \mid F_{t}\right] \\
& =\vartheta_{t} S_{t}+\delta_{t} B_{t}+E\left[C_{T}(\varphi)-C_{t}(\varphi) \mid F_{t}\right]=\vartheta_{t}^{\prime} S_{t}+\delta_{t}^{\prime} B_{t}
\end{aligned}
$$

Obviously, (13) satisfies definition 1, i. e., $\varphi^{\prime}=\left(\vartheta^{\prime}, \delta^{\prime}\right)$ is a strategy. By definition 2 and definition 3 , there are

$$
\begin{aligned}
& C_{T}\left(\varphi^{\prime}\right)-C_{t}\left(\varphi^{\prime}\right)=V_{T}\left(\varphi^{\prime}\right)-V_{t}\left(\varphi^{\prime}\right)-\sum_{j=t+1}^{T} \vartheta_{j-1}^{\prime} \Delta S_{j}-\sum_{j=t+1}^{T} \delta_{j-1}^{\prime} \Delta B_{j}+g \sum_{j=t+1}^{T} S_{j}\left|\Delta \vartheta_{j}^{\prime}\right| \\
& =V_{T}(\varphi)-V_{t}(\varphi)-E\left[C_{T}(\varphi)-C_{t}(\varphi) \mid F_{t}\right]-\sum_{j=t+1}^{T} \vartheta_{j-1} \Delta S_{j}-\sum_{j=t+1}^{T} \delta_{j-1} \Delta B_{j}+g \sum_{j=t+1}^{T} S_{j}\left|\Delta \vartheta_{j}\right| \\
& =C_{T}(\varphi)-C_{t}(\varphi)-E\left[C_{T}(\varphi)-C_{t}(\varphi) \mid F_{t}\right]
\end{aligned}
$$

and 


$$
\begin{aligned}
R_{t}\left(\varphi^{\prime}\right) & =E\left[\left(C_{T}\left(\varphi^{\prime}\right)-C_{t}\left(\varphi^{\prime}\right)\right)^{2} \mid F_{t}\right]=E\left\{\left[C_{T}(\varphi)-C_{t}(\varphi)-E\left[C_{T}(\varphi)-C_{t}(\varphi) \mid F_{t}\right]\right]^{2} \mid F_{t}\right\} \\
& =\operatorname{Var}\left[C_{T}(\varphi)-C_{t}(\varphi) \mid F_{t}\right] \leq E\left[\left(C_{T}(\varphi)-C_{t}(\varphi)\right)^{2} \mid F_{t}\right]=R_{t}(\varphi)
\end{aligned}
$$

i.e., $R_{t}\left(\varphi^{\prime}\right) \leq R_{t}(\varphi)$.

However, $\varphi=(\vartheta, \delta)$ is a risk-minimizing strategy, then, $R_{t}\left(\varphi^{\prime}\right) \geq R_{t}(\varphi)$.

Thus,

$$
\begin{aligned}
& R_{t}\left(\varphi^{\prime}\right) \\
& =E\left[\left(C_{T}\left(\varphi^{\prime}\right)-C_{t}\left(\varphi^{\prime}\right)\right)^{2} \mid F_{t}\right] \\
& =E\left\{\left[C_{T}(\varphi)-C_{t}(\varphi)-E\left[C_{T}(\varphi)-C_{t}(\varphi) \mid F_{t}\right]\right]^{2} \mid F_{t}\right\} \\
& =E\left[\left(C_{T}(\varphi)-C_{t}(\varphi)\right)^{2} \mid F_{t}\right] \\
& =R_{t}(\varphi)
\end{aligned}
$$

So, $E\left[\left(C_{T}(\varphi)-C_{t}(\varphi)\right) \mid F_{t}\right]=0$, i.e., $E\left[\left(C_{T}(\varphi) \mid F_{t}\right]=C_{t}(\varphi)\right.$,by definition $5, \varphi=(\vartheta, \delta)$ is a mean-self-financing strategy.

Proposition 2

Let $\varphi$ be a risk-minimizing strategy, and define $\hat{R}_{t}(\varphi):=\min _{\left(\varphi_{t}, \cdots, \varphi_{T}\right)} E\left[\left(C_{T}(\varphi)-C_{t}(\varphi)\right)^{2} \mid F_{t}\right]$, then

$$
\hat{R}_{t}(\varphi)=\min _{\varphi_{t}} E\left[\left(C_{t+1}(\varphi)-C_{t}(\varphi)\right)^{2} \mid F_{t}\right]+E\left(\hat{R}_{t+1}(\varphi) \mid F_{t}\right)
$$

Proof

Firstly, because

$$
\begin{aligned}
E & {\left[\left(C_{T}(\varphi)-C_{t}(\varphi)\right)^{2} \mid F_{t}\right] } \\
= & E\left\{\left[C_{T}(\varphi)-C_{t+1}(\varphi)+C_{t+1}(\varphi)-C_{t}(\varphi)\right]^{2} \mid F_{t}\right\} \\
= & E\left[\left(C_{T}(\varphi)-C_{t+1}(\varphi)\right)^{2} \mid F_{t}\right]+E\left[\left(C_{t+1}(\varphi)-C_{t}(\varphi)\right)^{2} \mid F_{t}\right] \\
& +2 E\left[\left(C_{T}(\varphi)-C_{t+1}(\varphi)\right)\left(C_{t+1}(\varphi)-C_{t}(\varphi)\right) \mid F_{t}\right]
\end{aligned}
$$

According to proposition $1, E\left[\left(C_{T}(\varphi) \mid F_{t}\right]=C_{t}(\varphi)\right.$, i.e., $C_{t}(\varphi)$ is a martingale, then, $C_{T}(\varphi)-C_{t+1}(\varphi)$ is independent of $C_{t+1}(\varphi)-C_{t}(\varphi)$, so, the last term in (15) equals 0 ,

Thus,

$$
\begin{aligned}
& \hat{R}_{t}(\varphi)=\min _{\left(\varphi_{t}, \cdots, \varphi_{T}\right)} E\left[\left(C_{T}(\varphi)-C_{t}(\varphi)\right)^{2} \mid F_{t}\right] \\
& =\min _{\left(\varphi_{t}, \cdots, \varphi_{T}\right)}\left\{E\left[\left(C_{T}(\varphi)-C_{t+1}(\varphi)\right)^{2} \mid F_{t}\right]+E\left[\left(C_{t+1}(\varphi)-C_{t}(\varphi)\right)^{2} \mid F_{t}\right]\right\} \\
& =\min _{\left(\varphi_{t}, \cdots, \varphi_{T}\right)} E\left\{E\left[\left(C_{T}(\varphi)-C_{t+1}(\varphi)\right)^{2} \mid F_{t+1}\right] \mid F_{t}\right\}+\min _{\left(\varphi_{t}, \cdots, \varphi_{T}\right)} E\left[\left(C_{t+1}(\varphi)-C_{t}(\varphi)\right)^{2} \mid F_{t}\right] \\
& =E\left\{\min _{\left(\varphi_{t+1}, \cdots, \varphi_{T}\right)} E\left[\left(C_{T}(\varphi)-C_{t+1}(\varphi)\right)^{2} \mid F_{t+1}\right] \mid F_{t}\right\}+\min _{\varphi_{t}} E\left[\left(C_{t+1}(\varphi)-C_{t}(\varphi)\right)^{2} \mid F_{t}\right] \\
& =\min _{\varphi_{t}} E\left[\left(C_{t+1}(\varphi)-C_{t}(\varphi)\right)^{2} \mid F_{t}\right]+E\left[\hat{R}_{t+1}(\varphi) \mid F_{t}\right]
\end{aligned}
$$

Proposition 3

As for $t=0,1, \cdots, T-1$, the risk-minimizing strategies for optimizing problem (8) are just as following 


$$
\left\{\begin{array}{l}
\vartheta_{t}^{*}=\frac{E\left(V_{t+1} S_{t+1} \mid F_{t}\right)-E\left(S_{t+1} \mid F_{t}\right) E\left(V_{t+1} \mid F_{t}\right)}{\operatorname{Var}\left(S_{t+1} \mid F_{t}\right)} \\
\delta_{t}^{*}=\frac{E\left(V_{t+1} \mid F_{t}\right)-\vartheta_{t}^{*} E\left(S_{t+1} \mid F_{t}\right)}{B_{t+1}}
\end{array}\right.
$$

Where

$$
\left\{\begin{array}{l}
V_{t}=\vartheta_{t} S_{t}+\delta_{t} B_{t}, t=0, \cdots, T-1 \\
V_{T}=H_{T}=\left(S_{T}-S_{0} \frac{I_{T}}{I_{0}}\right)^{+}
\end{array}\right.
$$

Proof

According to proposition $2, \hat{R}_{t}(\varphi)=\min _{\varphi_{t}} E\left[\left(C_{t+1}(\varphi)-C_{t}(\varphi)\right)^{2} \mid F_{t}\right]+E\left(\hat{R}_{t+1}(\varphi) \mid F_{t}\right)$, and refer to Poulsen et al(2009), $\hat{R}_{t+1}(\varphi)$ is independent of $\varphi_{t}=\left(\vartheta_{t}, \delta_{t}\right)$,so, the optimal strategy $\varphi_{t}^{*}=\left(\vartheta_{t}^{*}, \delta_{t}^{*}\right)$ may be acquired by minimizing $E\left[\left(C_{t+1}(\varphi)-C_{t}(\varphi)\right)^{2} \mid F_{t}\right]$.and (6) points to $C_{t+1}(\varphi)-C_{t}(\varphi)=V_{t+1}(\varphi)-\vartheta_{t} S_{t+1}-\delta_{t} B_{t+1}$, thus, solving the optimizing problem as following

$$
\min _{\varphi_{t}} E\left[\left(C_{t+1}(\varphi)-C_{t}(\varphi)\right)^{2} \mid F_{t}\right]=E\left[\left(V_{t+1}(\varphi)-\vartheta_{t} S_{t+1}-\delta_{t} B_{t+1}\right)^{2} \mid F_{t}\right]
$$

Which gives the optimal strategy at time $t$ just as (16).

Denote $\phi:=E\left(e^{y_{t}}\right) \quad, \quad \psi:=\operatorname{Var}\left(e^{y_{t}}\right), \quad R:=e^{r}$, where $r$ denotes riskless interest rate.

Let $\sum_{j=1}^{0} y_{j}=0, a_{11}=\frac{1}{\psi}, a_{10}=-\frac{\phi}{\psi}, b_{11}=-\frac{\phi}{R \psi}, b_{10}=\frac{\psi+\phi^{2}}{R \psi}$,by (16), under the constraint of $V_{T}=H_{T}=\left(S_{T}-S_{0} \frac{I_{T}}{I_{0}}\right)^{+}$, there is

$$
\left\{\begin{array}{l}
\vartheta_{T-n} S_{T-n}=\sum_{i=0}^{n}\left[a_{n i} E\left(H_{T} e^{\sum_{j=1}^{i} y_{T-n+j}} \mid F_{T-n}\right)\right] \\
\delta_{T-n} B_{T-n}=\sum_{i=0}^{n}\left[b_{n i} E\left(H_{T} e^{\sum_{j=1}^{i} y_{T-n+j}} \mid F_{T-n}\right)\right]
\end{array}\right.
$$

Where

$$
\begin{aligned}
& \left\{\begin{array}{l}
a_{n n}=\frac{1}{\psi}\left(a_{n-1, n-1}+b_{n-1, n-1}\right) \\
a_{n i}=-\frac{\phi}{\psi}\left(a_{n-1, i}+b_{n-1, i}\right)+\frac{1}{\psi}\left(a_{n-1, i-1}+b_{n-1, i-1}\right) \quad(i=1, \cdots, n-1) \\
a_{n 0}=-\frac{\phi}{\psi}\left(a_{n-1,0}+b_{n-1,0}\right)
\end{array}\right. \\
& \left\{\begin{array}{l}
b_{n n}=-\frac{\phi}{R \psi}\left(a_{n-1, n-1}+b_{n-1, n-1}\right) \\
b_{n i}=\frac{\psi+\phi^{2}}{R \psi}\left(a_{n-1, i}+b_{n-1, i}\right)-\frac{\phi}{R \psi}\left(a_{n-1, i-1}+b_{n-1, i-1}\right) \quad(i=1, \cdots, n-1) \\
b_{n 0}=\frac{\psi+\phi^{2}}{R \psi}\left(a_{n-1,0}+b_{n-1,0}\right)
\end{array}\right.
\end{aligned}
$$




\subsection{The Total Hedging Cost}

The total hedging cost, $T F=C_{T}(\varphi)+F_{T}(\varphi)$, consists of held position cost and transaction fee as (21) and (22)

$$
\begin{gathered}
C_{T}(\varphi)=V_{T}(\varphi)-\sum_{j=1}^{T} \vartheta_{j-1} \Delta S_{j}-\sum_{j=1}^{T} \delta_{j-1} \Delta B_{j} \\
F_{T}(\varphi)=\left|\vartheta_{0}\right| S_{0} f+\sum_{t=1}^{T-1}\left(\left|\vartheta_{t}-\vartheta_{t-1}\right| S_{t} f\right)+\left|\vartheta_{T-1}\right| S_{T} f
\end{gathered}
$$

Where $f$ denotes the transaction fee rate.

\section{Numerical Example}

In this paper, as an example, we assume that an investor has written European indexed stock options on stock of the Industrial and Commercial Bank of China (ab., ICBC, coded 601398) in China Stock Markets, and she attempts to hedge for this option with the underlying risky asset and the other riskless asset. The exercise price of indexed stock option is benchmarked against the terminal price of Shanghai Security Complex Index (ab., SSCI, coded 000001). We sampled high frequency history data of Shanghai Security Complex Index and stock ICBC and estimate the jump-diffusion process's parameters by MLE method, the estimated results are expressed in table 1.Morever, we set the riskless interest rate to be $0.36 \%$ which is offered by PBC (www.pbc.gov.cn). The price trends of the stock ICBC (601398) and SSCI (000001) during the lifetime of the hedged option are respectively shown in figure 1 and figure 2 .

Suppose an investor has written two shares of indexed stock options with half-year (1-year, respectively) maturity horizon on the stock ICBC in January 2009(July 2009, respectively), these two kinds of options all will expire in December 2009. The issuer hedges for her written options by minimizing the conditional mean square error of hedging cost process, i.e., $\min _{\varphi} E\left[\left(C_{T}(\varphi)-C_{t}(\varphi)\right)^{2} \mid F_{t}\right]$, under the constraint of $V_{T}(\varphi)=H_{T}=\left(S_{T}-K_{T}\right)^{+}$, where $K_{T}=S_{0} I_{T} / I_{0}$. and the strategy adjusting frequency is fixed to be 1-week(2-week,1-month, respectively). The strategy position trends are respectively shown in figure 3 and figure 4 with different strategy adjusting frequencies.

\section{Inert Figure 1 and Figure 2 - Here}

Firstly, as a whole, we can see from figure 1 that the stock price rose during 2009, with exceptional interval from July to September. Especially during the first half year of 2009, the stock price rose from 3.6CNY in Jan. up to its highest position 5.56CNY in June, subsequently fell at 5.44CNY in Dec., Similarly, the price of SSCI rose from 1800 points in Jan up to 3500 points in July, subsequently fluctuated around 3200 points and closed at 3277 points at the end of 2009. the price trends of two assets indicate that $S_{0}=3.6 C N Y$ and $I_{0}=1880$ points (respectively, $S_{0}=5.48 C N Y, I_{0}=3008$ points) for 1-year (respectively, half-year) maturity horizon indexed stock option, and $S_{T}=5.44 C N Y, I_{T}=3277$ points for two kinds of options, thus, according to $\left(S_{T}-S_{0} I_{T} / I_{0}\right)^{+}$, the terminal valuation of options all are 0 .

Secondly, as for hedging, It is so in theory that the father the maturity date is, the more violent the price fluctuates and the more the underlying asset should be held. In fact, from figure 3 and figure 4, one can see that more underlying asset is held for 1-year maturity horizon than that of half-year maturity horizon, and for two kinds of hedging, the underlying asset position all decrease with closing to maturity date, which indicates the correlation between length of maturity horizon and size of risk.

Insert Figure 3 and Figure 4 - Here

Furthermore, one may observe that the highest position of underlying asset for 1-year maturity horizon hedging is about 0.6 shares of stock when 0.09 shares for half-year maturity horizon hedging, there is obvious difference. Really, it is not surprising, because the underlying asset position to be held is also dependent of option's value in addition to maturity horizon, and for indexed stock option, it values $\left(S_{T}-S_{0} I_{T} / I_{0}\right)^{+}$. However, figure 1 and figure 2 indicate that, during the first half year of 2009, the stock price rose more than the Shanghai Securities Composite Index, moreover, the price of SSCI fell about 15\% during February and March while the price of stock ICBC fell only $7.5 \%$. thus, according to the price trend during the first half year, it is more possible for options to be in-the-money valuation state, and more underlying asset should be held to hedge for in-the-money 
option. In fact, the hedging positions of two options are closed to each other after the issue of half-year maturity horizon option.

Lastly, the total hedging cost for different kinds of hedging are shown in table 2, table 3 and table 4 .

Insert Table 2, Table 3 and Table 4 - Here

In table 2-4, there are negative hedging cost for 1-year maturity horizon hedging no matter what the strategy adjusting frequency and the transaction fee rate are, which indicates that there is some profit. However, there is a small quantity of expenses for half-year maturity horizon hedging. This is not paradoxical, because the foregoing price trend analysis tells that the terminal value of two options all equal 0,i.e., $H_{T}=\left(S_{T}-S_{0} I_{T} / I_{0}\right)^{+}=0$, thus, under the constraint of $V_{T}=H_{T}$, there is $C_{T}(\varphi)=-\sum_{j=1}^{T} \vartheta_{j-1} \Delta S_{j}-\sum_{j=1}^{T} \delta_{j-1} \Delta B_{j}$, which amounts to the position cost to be held and where there is $\Delta B_{j}>0$, so, there maybe $C_{T}(\varphi)<0$ (there is some profit) only if the stock price rise. As a matter of fact, figure 1 shows the rising trend of stock price. Taking hedging with weekly strategy adjusting frequency and 1-year maturity horizon as an example, during the first half year, there are 19 price rising chance versus 7 price descending chance among 26 strategy adjusting moments and the rising magnitude of stock price is greater than the range of a price drop, as a whole, the stock price rise from 3.6CNY at the beginning of January up to $5.56 \mathrm{CNY}$ at the end of June, which results in $0.3504 \mathrm{CNY}$ profit for holding long position underlying asset and $0.0082 \mathrm{CNY}$ transaction fee for adjusting position. Whereas, during the last half year, there are 11 price descending chance versus 15 price rising chance among 26 strategy adjusting moments and the rising magnitude of stock price is smaller than the range of a price drop, which results in 0.037CNY deficit for holding long position underlying asset and $0.00092 \mathrm{CNY}$ transaction fee for adjusting position, as a result, there is $0.3372 \mathrm{CNY}$ profit consisting of $0.3043 \mathrm{CNY}$ profit coming from holding risky asset and $0.0329 \mathrm{CNY}$ profit coming from riskless asset. However, as to half-year maturity horizon hedging, there are $0.0322 \mathrm{CNY}$ position deficit and $0.00178 \mathrm{CNY}$ transaction fee, deducting the profit coming from holding riskless asset, the total hedging cost amounts to $0.0252 \mathrm{CNY}$.

Once more, table2-table4 indicate that, for all kinds of hedging, the minimal total cost corresponds with twice weekly strategy adjusting frequency, i.e., higher adjusting frequency is not always more excellent. Higher strategy adjusting frequency may expend more for the existence of transaction fee, while lower strategy adjusting frequency may not hedge market risk. In a word, investors should decide strategy adjusting frequency according to all kinds of conditions such as maturity date and transaction fee rate, etc.

\section{Discussion and Conclusion}

Under the constraint of $V_{T}=H_{T}$, in this paper, we assume that the underlying asset price evolves as a jump-diffusion process and study the risk-minimizing problem of hedging indexed stock option. By solving optimization problem (8), we firstly give the explicit optimal strategy at some fixed position adjusting moment during option's maturity horizon. Then, numerical example results indicate that our technology is reasonable and feasible and several pieces of advices are presented as following

1) For hedging with longer maturity horizon, one may increase underlying asset position to cope with market fluctuating.

2) Results in table 2-table 4 indicate that advisable portfolio position may hedge financial market risk and reduce possible loss. Lower position adjusting frequency can not accord with market fluctuating and results in some loss, but excessively frequent position adjusting may also squander money for existence of commission fee.

In conclusion, because the financial market is protean, it is necessary to adjust hedging positions with excellent strategy scheme in order to get satisfactory hedging efficiency.

However, this study has several limitations which are also our future research directions. First, the acquired conclusions are subject to the limitation that our numerical example about the risk-minimizing hedging for indexed stock options was only based on a selected period of Chinese stock market, as to oversea stock market, 
we didn't present a demonstration example. Second, about hedging for traditional option, many literatures studied risk-minimizing hedging considering transaction fee, in this paper, as a try of hedging for indexed stock option, we did not consider transaction commission in optimizing model, however, which merits further investigation in future research. Besides above limitations of this study that need to be indicated, in this paper, we studied risk-minimizing hedging with fixed strategy adjusting moments during option's expiry, further research such as how to theoretically determine the strategy adjusting frequency for dynamic hedging is practically significant.

\section{Acknowledgements}

This research is supported by Shanghai Philosophy \& Social Science Planning Project (2009BJB001) and Shanghai Leading Academic Discipline Project (S30501).

\section{References}

Akhigbe A, Madura J \& Tucker A. (1996). Market-controlled Stock Options: A New Approach to Executive Compensation. Journal of Applied Corporate Finance, 9, 93-98. http://dx.doi.org/10.1111/j.1745-6622.1996.tb00106.x

Black F., \& Scholes M. (1973).The Pricing of Options and Corporate Liabilities. The Journal of Political Economy, 81(3), 637-654. [Online] Available: http://www.jstor.org/stable/1831029

Calvet AL., \& Rahman AH. (2006). The Subjective Valuation of Indexed Stock Options and Their Incentive Effects. Financial Review, 41(2), 205-227. http://dx.doi.org/10.1111/j.1540-6288.2006.00139.x

Chen Guan-ju, Feng Zong-xian \& Mu Gi-feng. (2002). Model and Application of Indexed Stock Options Based on Incentive Mechanism. Chinese Journal of Management Science, 10(3), 33-37.

Coleman, Thomas F., Li, Yuying \& Patron, M. (2003). Discrete hedging under piecewise linear risk minimization. The Journal of Risk, 5(3), 39-65.

Cyrus A. Ramezani, \& Yong Zeng. (2007). Maximum Likelihood Estimation of the Double Exponential Jump-Diffusion Process. Annals of Finance, 3(4), 487-507. http://dx.doi.org/10.1007/s10436-006-0062-y

Follmer H \& Sondermann D. (1986). Hedging of Non-Redundant Contingent Claims, in: Contributions to Mathematical Economics, eds. W. Hildenbrand and A.Mas-Colell, North-Holland, 205-223.

Follmer H., and Schweizer M. (1989). Hedging by Sequential Regression: An Introduction to the Mathematics of Option Trading. ASTIN Bulletin, 19, 29-42. http://dx.doi.org/10.2143/AST.19.3.2014900

Jin-Chuan Duan and Jason Wei. (2005). Executive Stock Options and Incentive Effects due to Systematic Risk. Journal of Banking \& Finance, 29(5),1185-1211. http://dx.doi.org/10.1016/j.jbankfin.2004.05.031

Møller, T. (2004). Risk-Minimization, in J. Teugels \& B. Sundt, eds. Encyclopedia of Actuarial Science, 3, 1501-1508.

Poulsen R., K.R. Schenk-Hoppé, \& C. Ewald. (2009). Risk Minimization in Stochastic Volatility Models: Model Risk and Empirical Performance. Quantitative Finance, 9(6), 693-704. http://dx.doi.org/10.1080/14697680902852738

Schweizer, M. (1991). Option Hedging for Semimartingales. Stochastic Processes and their Applications, 37(2), 339-363. http://dx.doi.org/10.1016/0304-4149(91)90053-F

Schweizer, M. (1995). On the minimal martingale measure and the Follmer-Schweizer decomposition. Stochastic Analysis and Applications, 13, 573-599. http://dx.doi.org/10.1080/07362999508809418

Schweizer, M. (1999). A guided tour through quadratic hedging approaches, in Option Pricing, Interest Rates and Risk Management, eds. J.Jouini, M.Cvitanic, M.Musiela, Cambridge University Press, 538-574.

Shane A. Johnson \& Yisong S. Tian. (2000). Indexed Executive Stock Options. Journal of Financial Economics, 57(1), 35-64. http://dx.doi.org/10.1016/S0304-405X(00)00050-7

Schiefner L. (2002). Risk-Minimizing Hedging of General Cash Flows in Discrete Time. [Online] Available: http://ssrn.com/abstract=302320

Ubelhart M. (1981). A New Look at Executive Compensation Plans. Cash Flow, 2, 20-24.

Zhong Mei-rui \& Huang Jian-bai. (2005). The Application of Relative Performance and Portfolio Idea in Option Pay Design. Chinese Journal of Management Science, 13(2), 130-136. 
Table 1. The MLE Valuations of Parameters

\begin{tabular}{|l|c|c|c|c|c|}
\hline parameters & $\mu\left(\mu^{(I)}\right)$ & $\sigma\left(\sigma^{(I)}\right)$ & $\mu_{J}\left(\mu_{J}^{(I)}\right)$ & $\sigma_{J}\left(\sigma_{J}^{(I)}\right)$ & $\lambda\left(\lambda^{(I)}\right)$ \\
\hline Underlying tock(S) & 0.0017 & 0.0179 & 0.0214 & 0.0454 & 0.0292 \\
\hline Index(I) & 0.0023 & 0.019 & -0.0155 & 0.0519 & 0.0453 \\
\hline
\end{tabular}

Table 2. Total Hedging Cost with 1\%o Transaction Fee Rate

\begin{tabular}{|c|c|c|c|}
\hline maturity & weekly & Twice weekly & monthly \\
\hline 1-year maturity horizon & -0.3372 & -0.3745 & -0.1971 \\
\hline Half-year maturity horizon & 0.0252 & 0.0142 & 0.0151 \\
\hline
\end{tabular}

Table 3. Total Hedging Cost with 2\%o Transaction Fee Rate

\begin{tabular}{|c|c|c|c|}
\hline maturity & weekly & Twice weekly & monthly \\
\hline 1-year maturity horizon & -0.3326 & -0.3701 & -0.1952 \\
\hline Half-year maturity horizon & 0.0262 & 0.0147 & 0.0156 \\
\hline
\end{tabular}

Table 4. Total Hedging Cost with 4\%o Transaction Fee Rate

\begin{tabular}{|c|c|c|c|}
\hline maturity & weekly & Twice weekly & monthly \\
\hline 1-year maturity horizon & -0.3234 & -0.3613 & -0.1914 \\
\hline Half-year maturity horizon & 0.0280 & 0.0159 & 0.0167 \\
\hline
\end{tabular}

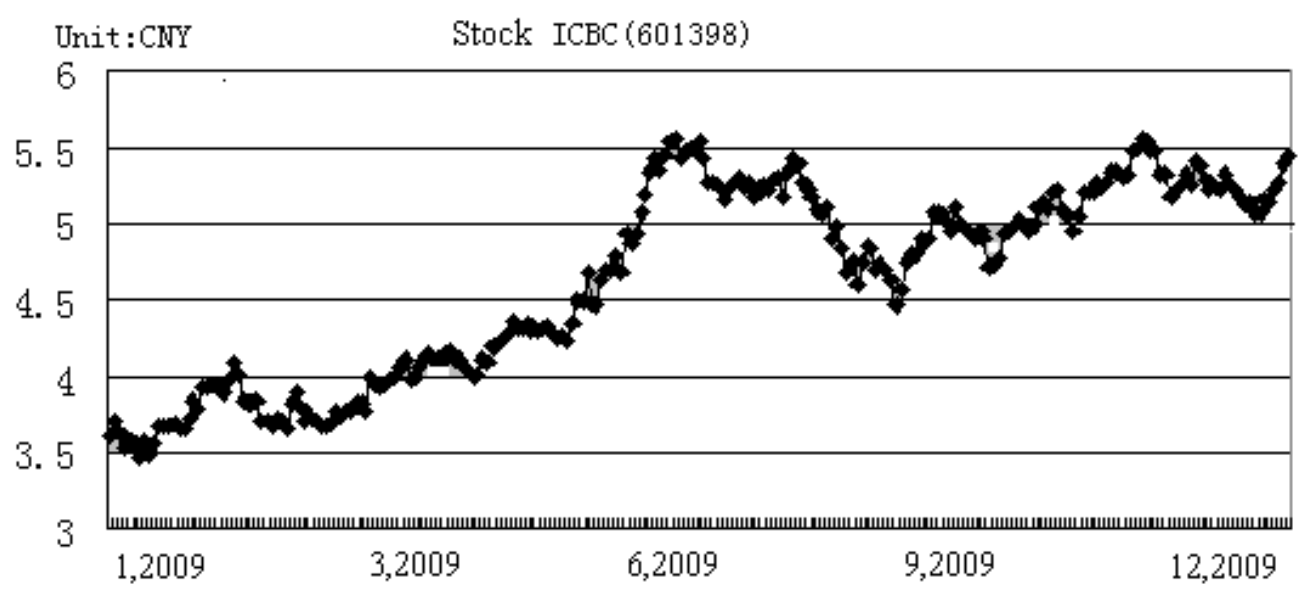

Figure 1. The Price Trend of Stock ICBC(601398) form Jan. $1^{\text {st }}, 2009$ to Dec.31th,2009 


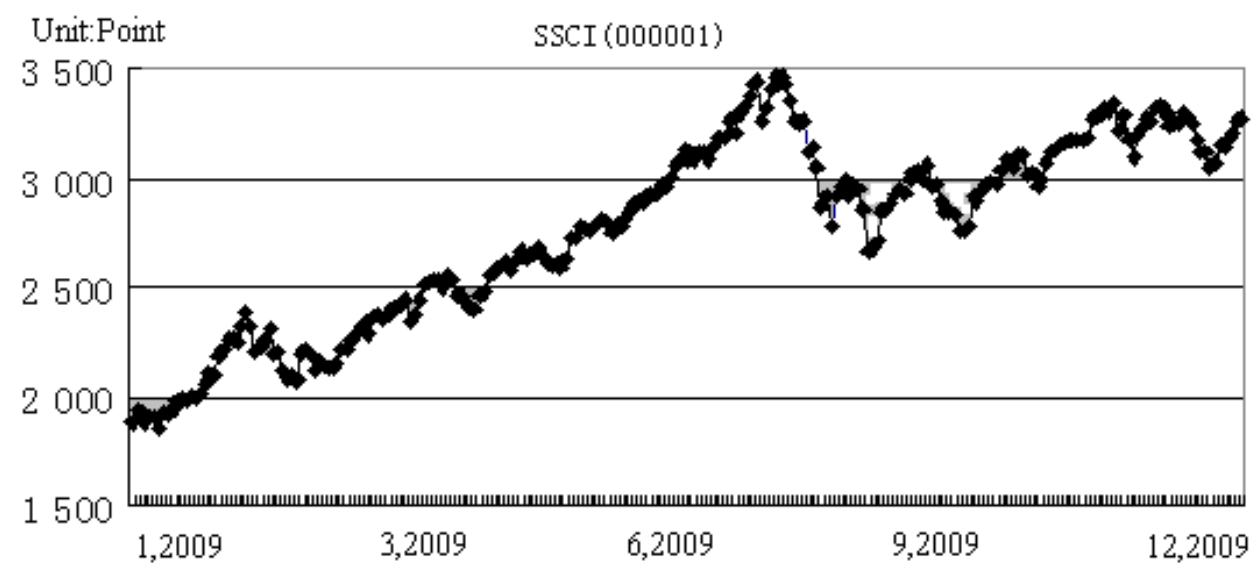

Figure 2. The Price Trend of SSCI(000001) from Jan. $1^{\text {st }}, 2009$ to Dec.31th,2009

Unit:Share Position plot of the underlying risky asset

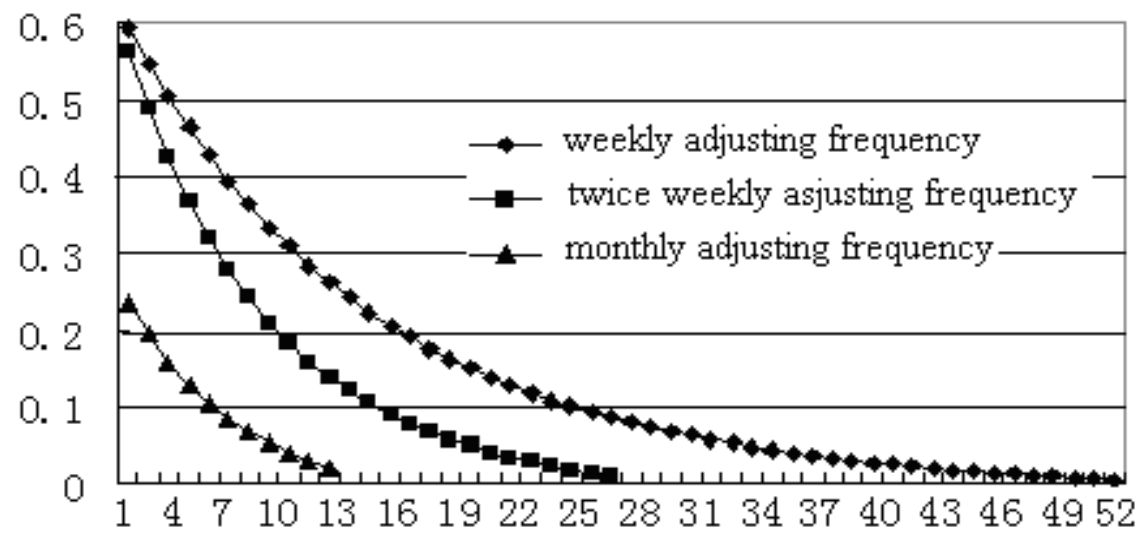

Figure 3. Plot of the Underlying Risky Asset Position for 1-year Maturity Horizon Hedging

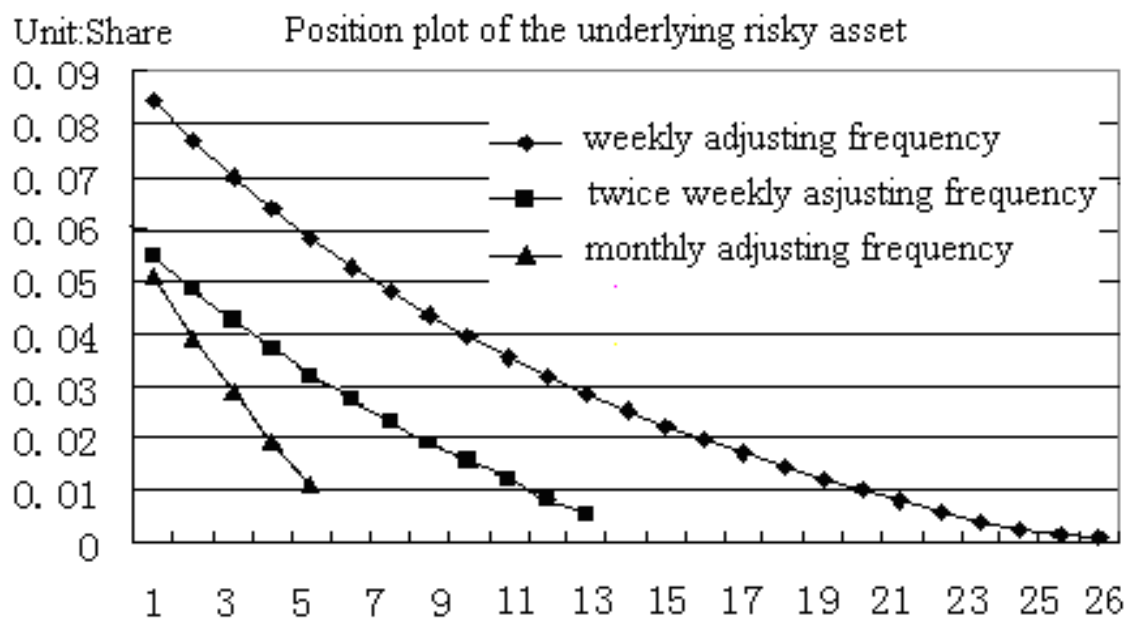

Figure 4. Plot of the Underlying Risky Asset Position for Half-year Maturity Horizon Hedging 